\title{
Plane Wave Analysis of Side Inlet/Outlet Chamber Mufflers with Mean Flow
}

\author{
M. L. Munjal \\ Center of Excellence for Technical Acoustics, Department of Mechanical Engineering, Indian \\ Institute of Science, Bangalore 560 012, India
}

\begin{abstract}
Integration of side inlet and/or side outlet expansion chambers into the rest of the muffler system for prediction of overall transmission loss (TL) or insertion loss requires knowledge of the four-pole parameters of these elements for propagation of plane waves in a moving medium. In this paper, transfer matrices have been derived for side inlet and side outlet elements for typically small mean flow Mach numbers. These are validated against the 3-D model existing in the literature for a stationary medium and for acoustically long chambers. The effect of mean flow on TL of the side inlet/ outlet chambers has been demonstrated. Incidentally, it has been shown that side inlets and side outlets behave more or less like extended inlets and extended outlets, respectively. (C) 1997 Elsevier Science Ltd.
\end{abstract}

Keywords: Mufflers, duct acoustics.

\section{NOMENCLATURE}

$d$ diameter of the chamber

$j \quad(-1)^{1 / 2}$

$k$ wave number

$K$ stagnation pressure loss factor

$l$ length

$M$ mean flow Mach number

$p$ pressure

$r$ radius of the inlet/outlet pipe

$R$ radius of the chamber 


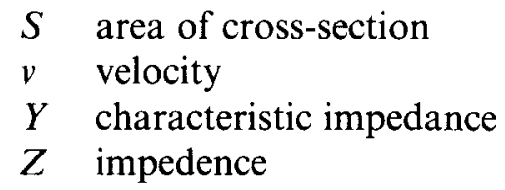

\section{Subscripts}

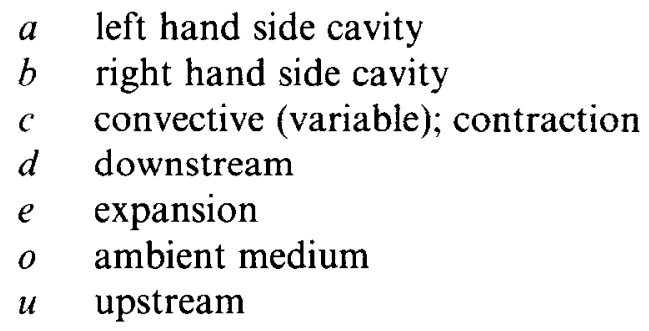

\section{INTRODUCTION}

Most mufflers have their inlet and outlet at the ends, with their axes parallel to that of the circular chamber. Analysis of these mufflers has been dealt with at length by the author ${ }^{1}$ among others. Sometimes however, for logistical reasons, the inlet and/or outlet are provided on the sides with their axes normal to the axis of the muffler chamber or shell, as shown in Fig. 1. End inlets or outlets being parallel to the axis of the chamber may also be called axial inlets or outlets. Axial inlets or outlets need not be co-axial with the chambers; an offset is often necessary or desirable. ${ }^{2-7}$ Believing that threedimensional effects (higher-order modes) would dominate in the case of side inlet/outlet chambers, $\mathrm{Yi}$ and Lee ${ }^{4,5}$ presented a three-dimensional analysis of such chambers for a stationary medium. Derivation of the four-pole parameters by means of their 3-D analytical technique is of general application, but may be time-consuming and cumbersome. Besides, the convective as well as dissipative effects of mean flow have been ignored in their analysis. For most engineering applications, noise is limited to low and medium frequencies where plane waves would dominate. Therefore, it is desirable to derive four-pole parameters for side inlet/outlet chambers considering pure plane waves and incompressible mean flow. This is what is attempted in the present Technical Note.

By making use of the equations of mass continuity, stagnation pressure loss and the end resonator impedance, transfer matrices have been derived here for typically low mean flow Mach numbers $\left(M^{2}<<1\right)$, for a side inlet junction and side outlet junction. These are then used to predict transmission loss (TL) for two configurations from Refs 3 and 4, for ready comparison at low and medium frequencies where plane waves are expected to predominate. 
(c)

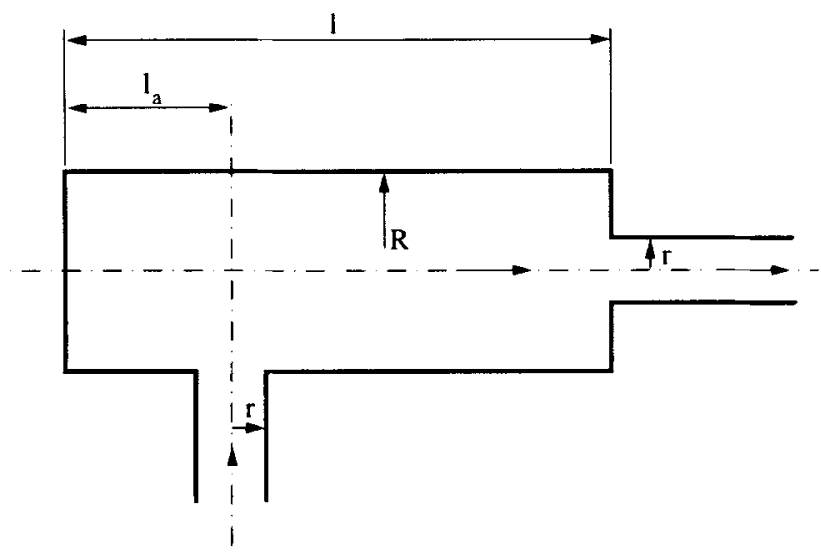

(b)

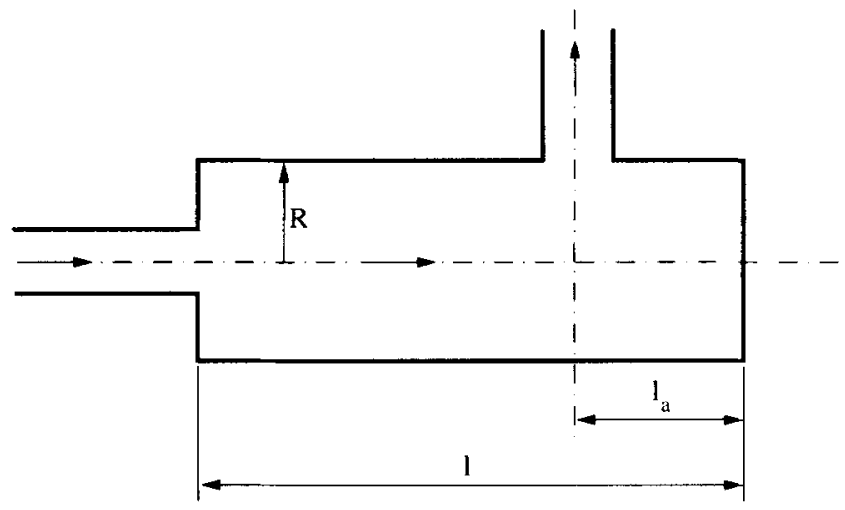

(c)

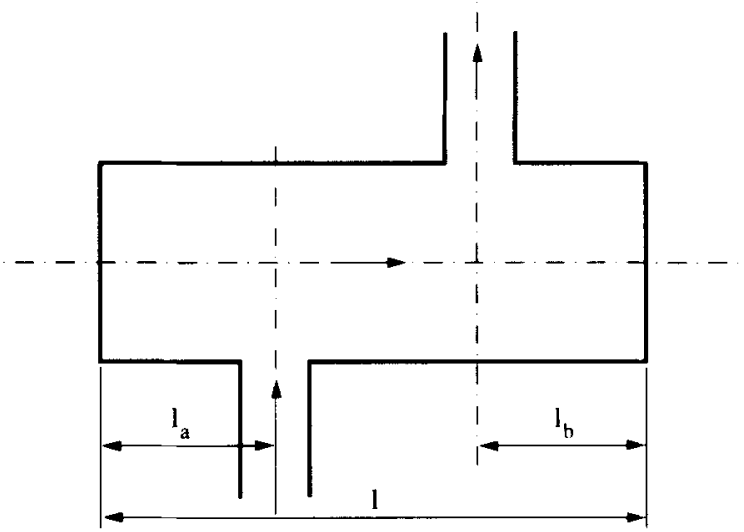

Fig. 1. Schematic diagrams of (a) side-inlet end-outlet chamber; (b) end-inlet side-outlet chamber and (c) side-inlet side-outlet chamber. 
The effect of mean flow on TL has been shown separately. Finally, it has been indicated that side inlet/outlet chambers behave more or less like extended inlet/outlet chambers, and therefore may be designed accordingly.

\section{TRANSFER MATRIX FOR SIDE INLET EXPANSION ELEMENT}

Let $u, d$ and $a$ denote upstream point, downstream point and the end-cavity resonator, respectively, in Fig. 2. These correspond to points 3,1 and 2 in the corresponding extended inlet element shown in Fig. 2.13 of Ref. 1. Neglecting $M^{2}$ with respect to unity, the stagnation pressure eqn ((3.81)) of Ref. 1 yields

$$
p_{c, u}=p_{c, d}+K_{e} M_{d} Y_{d} v_{c, d}, \quad K_{e}=\left[\left(S_{d} / S_{u}\right)-1\right]^{2}
$$
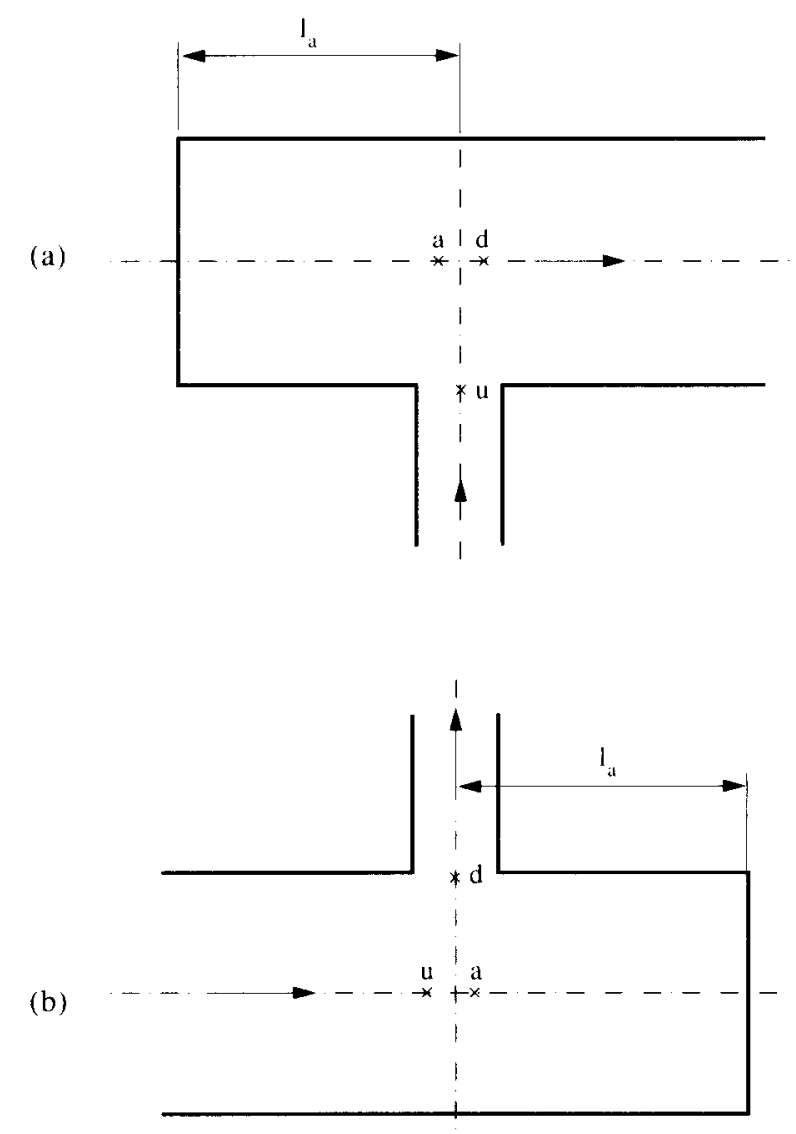

Fig. 2. Schematics for derivation of transfer matrices; (a) side inlet and (b) side outlet. 
and the mass continuity eqn (3.89) of Ref. 1 gives

$$
v_{c, u}=v_{c, d}+v_{a}
$$

where the convective state variables $p_{c}$ and $v_{c}$ at any point in a duct are related to the classical state variables $p$ and $v$ (at the same point) as ${ }^{1}$

$$
\left[\begin{array}{c}
p_{c} \\
v_{c}
\end{array}\right]=\left[\begin{array}{cc}
1 & M Y \\
\frac{M}{Y} & 1
\end{array}\right]\left[\begin{array}{l}
p \\
v
\end{array}\right]
$$

or conversely as

$$
\left[\begin{array}{l}
p \\
v
\end{array}\right]=\left[\begin{array}{cc}
1 & -M Y \\
-\frac{M}{Y} & 1
\end{array}\right]\left[\begin{array}{l}
p_{c} \\
v_{c}
\end{array}\right]
$$

where $M$ and $Y$ are the mean flow Mach number and characteristic impedance, respectively, and $M^{2}$ has been neglected with respect to unity.

It may be noted here that the loss factor $K_{e}$ in eqn (1) is for sudden expansion. In its current form, it is based on axial inlet and exit flow alignment and assumes reattachment of fluid to the bounding surfaces. It does not consider a change in the direction of flow, as in the case of a side inlet, and therefore is approximate at best.

For the end cavity closed at the other end by a rigid end plate, eqns (3.94) and (3.95) of Ref. 1 give

$$
p_{a} / v_{a}=Z_{a}=-j Y_{a} \cot \left(k_{o} 1_{a}\right)
$$

It may be observed from Fig. 2(a) that

$$
p_{a}=p_{d}
$$

Equations (1)-(6) may be seen to readily yield the transfer matrix relation

$$
\left[\begin{array}{c}
p_{c, u} \\
v_{c, u}
\end{array}\right]=\left[\begin{array}{cc}
1 & K_{e} M_{d} Y_{d} \\
\frac{1}{Z_{u}} & 1-\frac{M_{d} d_{d}}{Z_{u}}
\end{array}\right]\left[\begin{array}{c}
p_{c, d} \\
v_{c, d}
\end{array}\right]
$$

The corresponding transfer matrix relation in terms of the classical state variables can be obtained readily from eqns (3), (4) and (7):

$$
\left[\begin{array}{c}
p_{u} \\
v_{u}
\end{array}\right]=\left[\begin{array}{cc}
1 & -M_{u} Y_{u} \\
-\frac{M_{u}}{Y_{u}} & 1
\end{array}\right]\left[\begin{array}{cc}
1 & K_{e} M_{d} Y_{d} \\
\frac{1}{Z_{a}} & 1-\frac{M_{d} Y_{d}}{Z_{a}}
\end{array}\right]\left[\begin{array}{cc}
1 & M_{d} Y_{d} \\
M_{d} & 1
\end{array}\right]\left[\begin{array}{c}
p_{d} \\
y_{d}
\end{array}\right]
$$


Successive multiplication of the three matrices in eqn (8), and neglecting terms of the order of $M^{2}$ with respect to unity, yields

$$
\left[\begin{array}{c}
p_{u} \\
v_{u}
\end{array}\right]=\left[\begin{array}{cc}
1-\frac{M_{u} Y_{u}}{Z_{a}} & \left(1+K_{e}\right) M_{d} Y_{d}-M_{u} Y_{u} \\
\frac{1}{Z_{a}} & 1
\end{array}\right]\left[\begin{array}{c}
p_{d} \\
v_{d}
\end{array}\right]
$$

A sudden area discontinuity is analogous to a lumped inductive (inertive) impedance. Over the years, this impedance has been calculated by means of a three-dimensional analysis for co-axial discontinuities for stationary medium $^{8}$ and moving medium ${ }^{9}$ and, more recently, for offset inlet/outlet ${ }^{7}$ for stationary medium, valid for frequencies right up to the cut-on frequencies of the first higher order mode. Peat ${ }^{9}$ found that the effect of mean flow on both the real and imaginary parts of the discontinuity impedance is practically negligible. Discontinuities of the types shown in Fig. 2 have not been analysed so far for prediction of inertance due to evanescent higher order modes generated at the junction. However, its geometry is somewhat similar to a circular piston in an infinite baffle. Therefore, heuristically, adding lumped inertance due to 3-D effects for a piston in an infinite baffle at the first-row second-column location as in the transfer matrix of a lumped inline impedance, ${ }^{1}$ gives the required transfer matrix:

$$
\left[\begin{array}{cc}
1-\frac{M_{u} Y_{u}}{Z_{a}} & \left(1+K_{e}\right) M_{d} Y_{d}-M_{u} Y_{u}+Y_{u}\left(j 0.85 k_{o} r_{u}\right) \\
{\frac{1}{Z_{a}}}^{n_{a}} & 1
\end{array}\right]
$$

\section{TRANSFER MATRIX FOR THE SIDE OUTLET CONTRACTION ELEMENT}

Equations (2)-(5) would hold for the side-outlet contraction element (Fig. 2b) as well, except that the stagnation pressure loss factor would be given by

$$
K_{c}=\left(1-\frac{S_{d}}{S_{u}}\right) / 2
$$

It may be noted here that $k_{c}$ suffers from the same limitation as $k_{e}$, as remarked earlier following eqn (4). It has also been used for want of a more pertinent expression in the published literature.

Equation (6) is replaced by the equation (see Fig. 2b):

$$
p_{u}=p_{a}
$$


These equations lead to the following transfer matrix relation in terms of the convective state variables:

$$
\left[\begin{array}{c}
p_{c, u} \\
v_{c, u}
\end{array}\right]=\left[\begin{array}{cc}
1 & K_{c} M_{d} Y_{d} \\
\frac{1}{Z_{a}} & 1-\frac{M_{u} Y_{u}}{Z_{a}}
\end{array}\right]\left[\begin{array}{c}
p_{c, d} \\
v_{c, d}
\end{array}\right]
$$

The corresponding relationship in terms of the acoustic state variables may be obtained by means of the transformation eqns (3) and (4) as in eqn (8). Neglecting terms of the order of $M^{2}$ with respect to unity, and again noting that

$$
\frac{M_{d}}{Y_{d}}=\frac{M_{u}}{Y_{u}}
$$

one obtains

$$
\left[\begin{array}{c}
p_{u} \\
v_{u}
\end{array}\right]=\left[\begin{array}{cc}
1-\frac{M_{u} Y_{u}}{Z_{a}} & \left(K_{c}+1\right) M_{d} Y_{d}-M_{u} Y_{u} \\
\frac{1}{Z_{a}} & 1+\frac{M_{d} Y_{d}-M_{u} Y_{u}}{Z_{a}}
\end{array}\right]\left[\begin{array}{c}
p_{d} \\
v_{d}
\end{array}\right]
$$

Finally, from heuristic reasoning as for the side inlet, adding inertive impedance due to 3-D effects at the first-row second-column location, one obtains the desired transfer matrix:

$$
\left[\begin{array}{cc}
1-\frac{M_{u} Y_{u}}{Z_{a}} & \left(K_{c}+1\right) M_{d} Y_{d}-M_{u} Y_{u}+j\left(0.85 k_{n} r_{d}\right) Y_{d} \\
\frac{1}{Z_{a}} & 1+\frac{M_{d} Y_{d}-M_{u} Y_{u}}{Z_{a}}
\end{array}\right]
$$

\section{RESULTS AND DISCUSSION}

Combining transfer matrices (10) and (16) for side inlets and side outlets, respectively, with those of other elements like uniform tubes, transmission loss can easily be calculated ${ }^{1}$ by means of an existing general user-friendly program in FORTRAN on a PC486 machine. For corroboration of the plane wave model with the 3-D model predictions and experimental observations of $\mathrm{Yi}$ and Lee, ${ }^{4,5} \mathrm{TL}$ was computed for the following configurations for a stationary medium.

Side inlet end outlet chamber (Fig. 1a):

$$
l=0.3 \mathrm{~m}, \quad R=0.061 \mathrm{~m}, \quad l_{a}=0.15 \mathrm{~m}, \quad R / r=5
$$


Side inlet side outlet chamber (Fig. 1c):

$$
l=0.3 \mathrm{~m}, \quad R=0.061 \mathrm{~m}, \quad l_{a}=0.15 \mathrm{~m}, \quad l_{b}=0.075 \mathrm{~m}, \quad R / r=5
$$

Figure 3 compares predictions of the present l-D model with those of the Yi and Lee's 3-D model ${ }^{4}$ for the side-inlet side-outlet configuration of Fig. 1c with the outlet rotated $\pi / 2$ radians with respect to the inlet, so as to avoid tunnelling of higher order modes. It may be noted that the plane wave model predictions are quite satisfactory for this acoustically long chamber $(l / d=2.46, d=2 R)$. The same may be noticed from Fig. 4 for the side-inlet end-outlet configuration of Fig. 1a, where Yi and Lee's 3-D model predictions and experimental observations ${ }^{5}$ are compared with the present $1-D$ model predictions. However, for the acoustically short chambers $(1<2 d)$, 3 -D effects (higher order modes) may be very strong and then the plane wave model presented here may lead to significant errors, particularly if the angular disposition of the outlet permits tunnelling of higher order modes. ${ }^{2,6}$

Incidentally, side inlet behaves quite like an extended inlet and side outlet behaves like an extended outlet, as shown in Fig. 5. Their TL values are compared in Fig. 6 for the side-inlet side-outlet configuration of Fig. 1c with

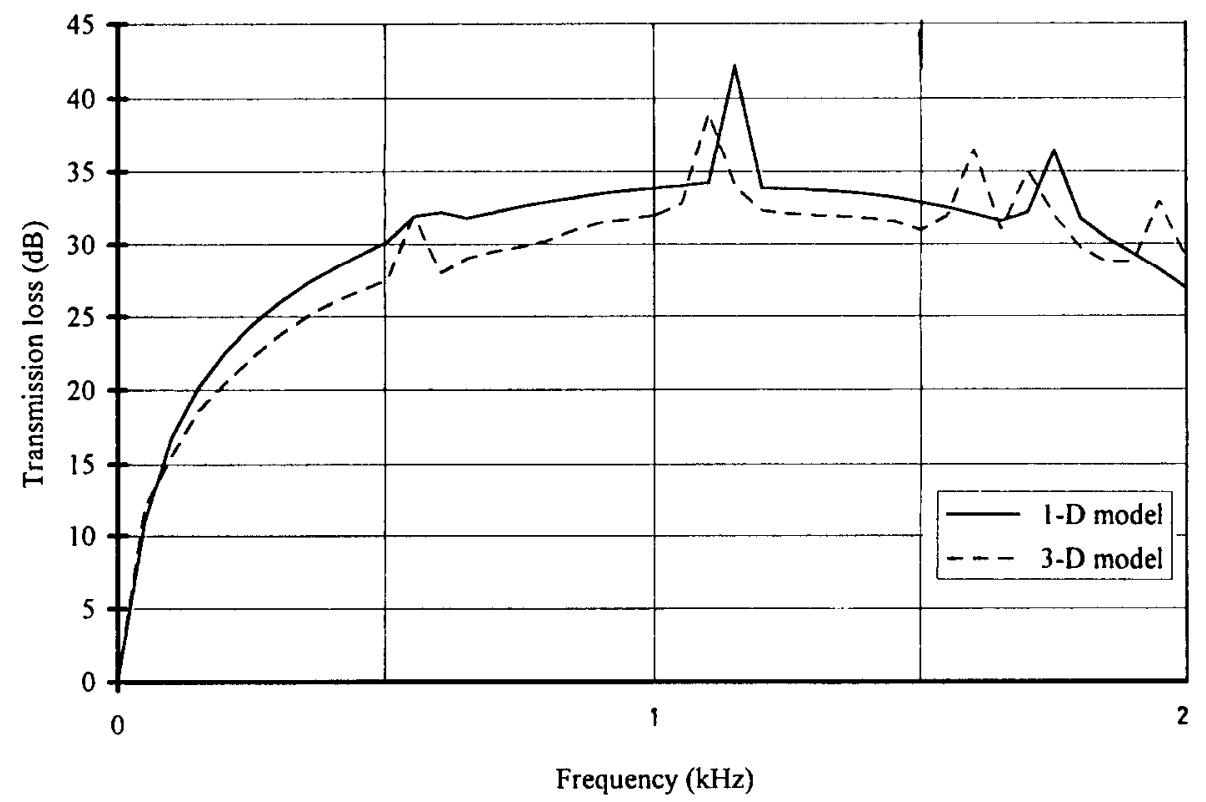

Fig. 3. Comparison of the present 1-D model with Yi and Lee's 3-D model for a side-inlet side-outlet chamber. 


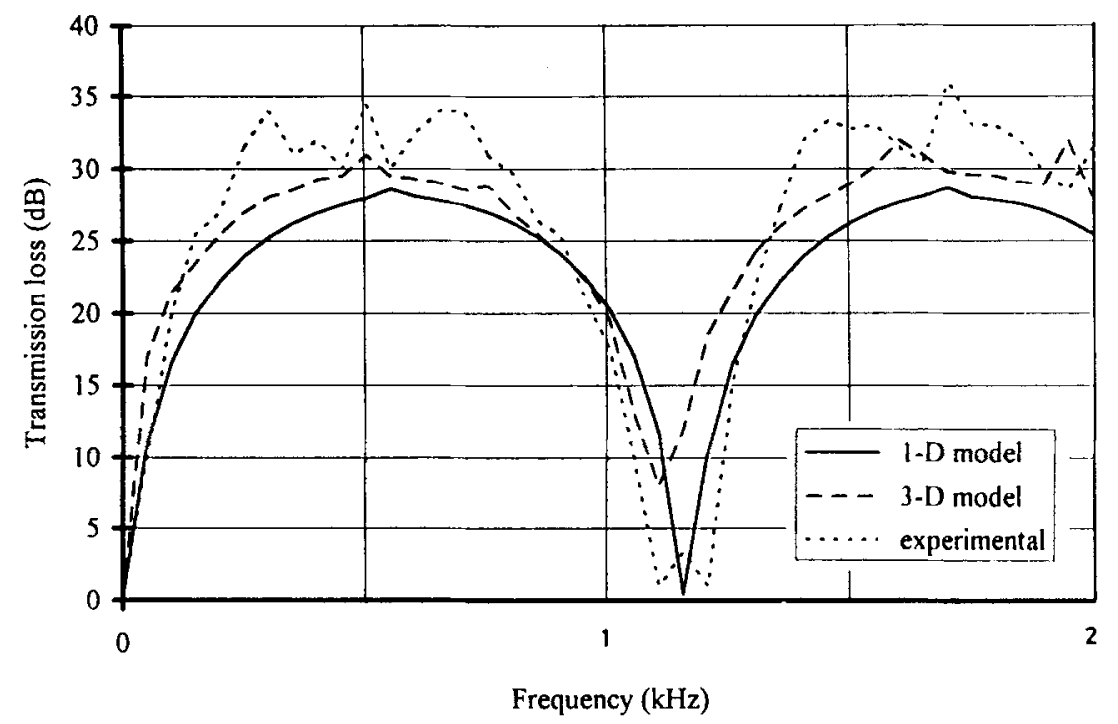

Fig. 4. Comparison of the present l-D model with Yi and Lee's 3-D model and experimental measurements for a side-inlet end-outlet chamber.

(a)

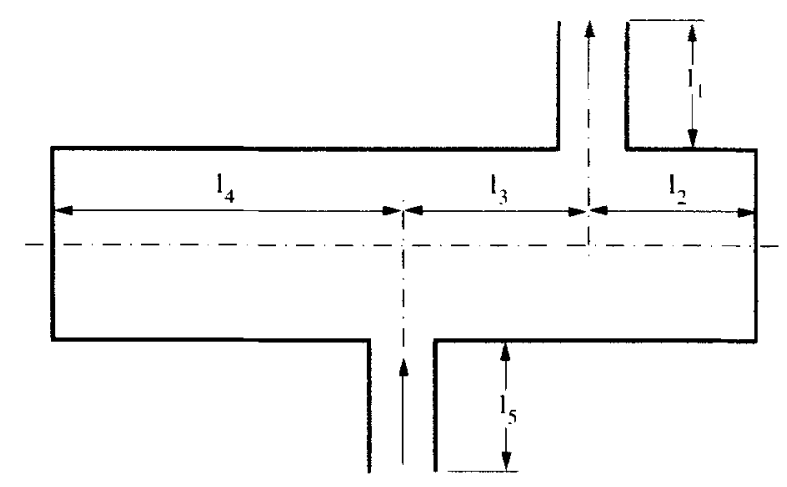

(b)

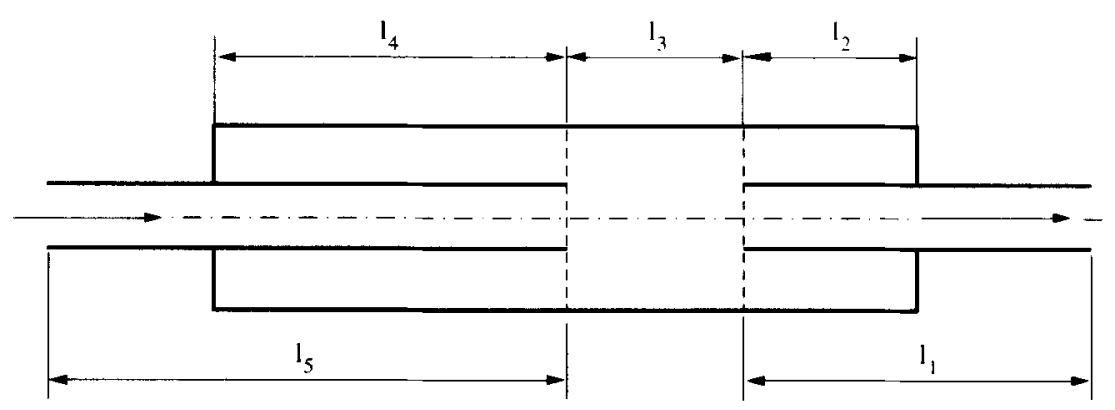

Fig. 5. Functional equivalence of (a) side-inlet side-outlet chamber and (b) extended-inlet extended-outlet chamber. 


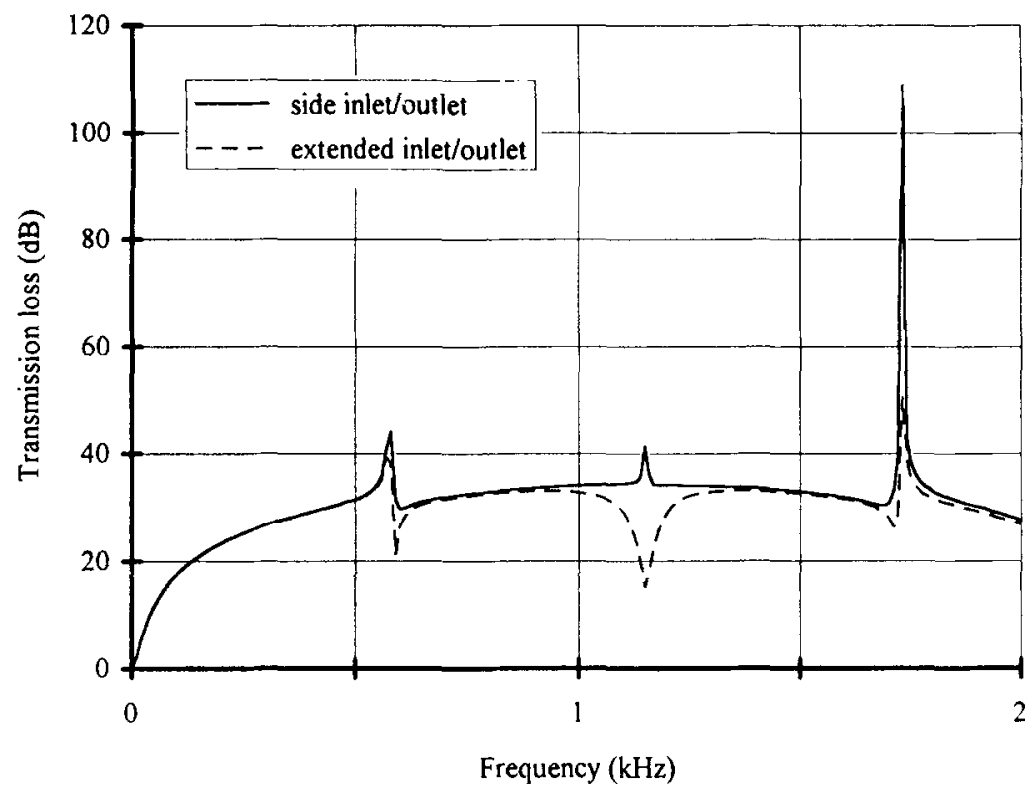

Fig. 6. Comparison of a side inlet/outlet chamber with the corresponding extended inlet/outlet chamber.

dimensions as in eqn (18) above and the mean flow Mach number in the pipe, $M=0.1$. The conceptual similarity may be seen to be reflected very well in the computed TL spectra as well.

It has been observed that the effect of mean flow on TL of the same sideinlet side-outlet configuration is minimal $( \pm 2 \mathrm{~dB}$ ) for typical Mach numbers $(M=0.1)$, and therefore has not been shown in this Technical Note. It indicates that the stationary-medium 3-D model of $\mathrm{Yi}$ and $\mathrm{Lee}^{4}$ may be used for moving medium as well with minimal errors.

\section{CONCLUSIONS}

The plane wave model presented here for side inlet/outlet chamber is much simpler and faster than the corresponding 3-D model. ${ }^{4,5}$ Besides, it incorporates the effect of mean flow, although the same has now been found to be practically negligible. Incidentally, it is shown that a side inlet behaves like an extended inlet and a side outlet behaves like an extended outlet. However, the 1-D model would work only for acoustically long chambers $(1 \geq 2 d)$ and for those angular dispositions of the outlet (with respect to the inlet) that do not allow tunnelling of higher order modes. ${ }^{2,6}$ This should be kept in mind while designing mufflers for medium and higher frequencies. 


\section{ACKNOWLEDGEMENT}

The author is grateful to the Department of Science and Technology of the Government of India for funding the Center of Excellence for Technical Acoustics, thereby enabling the research work reported here.

\section{REFERENCES}

1. Munjal, M. L., Acoustics of Ducts and Mufflers. John Wiley, New York, 1987.

2. Eriksson, L. J., Higher-order mode effects in circular ducts and expansion chambers. Journal of the Acoustical Society of America, 1980, 68, 545-550.

3. Ih, J.-G. and Lee, B.-H., Analysis of higher order mode effects in the circular expansion chamber with mean flow. Journal of the Acoustical Society of America, 1985, 77, 1377-1388.

4. Yi, S. I. and Lee, B. H., Three dimensional acoustic analysis of circular expansion chambers with a side inlet and side outlet. Journal of the Acoustical Society of America, 1986, 79, 1299-1306.

5. Yi, S. I. and Lee, B. H., Three dimensional acoustic analysis of circular expansion chambers with a side inlet and end outlet. Journal of the Acoustical Society of America, 1987, 81, 1279-1287.

6. Sahasrabudhe, A. D., Munjal, M. L. and Ramu, S. A., Design of expansion chamber mufflers incorporating 3-D effects. Noise Control Engineering Journal, 1992, 38, 27-38.

7. Sahasrabudhe, A. D., Munjal, M. L. and Ramu, S. A., Analysis of inertance due to the higher order mode effects in a sudden area discontinuity. Journal of Sound and Vibration, 1995, 185, 515-529.

8. Kergomard, J. and Garcia, A., Simple discontinuities in acoustic wave guides at low frequencies: Critical analysis and formulae. Journal of Sound and Vibration. 1987, 114, 467-479.

9. Peat, K. S., The acoustical impedance at discontinuities of ducts in the presence of a mean flow. Journal of Sound and Vibration, 1988, 127, 123-132. 